Pacific Journal of Mathematics

ON EXPANSIVE HOMEOMORPHISMS 


\title{
ON EXPANSIVE HOMEOMORPHISMS
}

\author{
B. F. BRYANT
}

1. Introduction. A homeomorphism $\phi$ of a compact metric space $X$ onto $X$ is said to be expansive provided there exists $d>0$ such that if $x, y \in X$ with $x \neq y$, then there exists an integer $n$ such that $\rho\left(x \phi^{n}, y \phi^{n}\right)>d$ (see [1] and [3]). The question arises as to the possibility of extending the results concerning expansive homeomorphisms to compact uniform spaces. The extension is possible, although trivial in light of the corollary to Theorem 1.

In $\S \S 3$ and 4 the setting is a compact metric space $X$. Theorem 2 is stronger than Theorem 10.36 of [1] in that we do not require $X$ to be self-dense. Also, the lemmas of which Theorem 2 is a consequence are perhaps of some interest in themselves. In $\S 4$ we show that if $X$ is self-dense, then for each $x \in X$ and each $\varepsilon>0$ there exists $y \in U(\varepsilon, x)$ such that $x$ and $y$ are not doubly asymptotic.

2. A homeomorphism $\phi$ of a compact uniform space $(X, \mathscr{U})$ onto $(X, \mathscr{C})$ is said to be expansive provided there exists $U \in \mathscr{U}$ such that $U \neq \Delta$ (the diagonal) and if $x, y \in X$ with $x \neq y$, then there exists an integer $n$ such that $\left(x \phi^{n}, y \phi^{n}\right) \notin \bar{U}$. For uniform spaces we use the notation of [2], but following Weil [4] we suppose $(X, \mathscr{U})$ is Hausdorff; i. e., $\cap\{U: U \in \mathscr{U}\}=\Delta$. We also suppose that each $U \in \mathscr{U}$ is symmetric.

THEOREM 1. Let $(X, \mathscr{U})$ be a compact uniform space which is not metrizable and let $\phi$ be a homeomorphism of $X$ onto $X$. If $U \in \mathscr{U}$, then there exist $x, y \in X$ with $x \neq y$ such that $\left(x \phi^{n}, y \phi^{n}\right) \in U$ for each integer n. (Compare with Theorem 10.30 of [1].)

Proof. Select $V \in \mathscr{U}$ such that $V \circ V \circ V \subset U$ and $\bar{V} \subset U$ (see [2], p. 180). Since $\phi^{n}$, for each integer $n$, is uniformly continuous, we may choose $U_{1} \in \mathscr{U}$ with $U_{1} \subset V$ such that $(p, q) \in U_{1}$ implies $\left(p \phi^{k}, q \phi^{k}\right) \in V$ for $k= \pm 1$. For $i>1$, choose $U_{i} \in \mathscr{U}$ with $U_{i} \subset U_{i-1}$ such that $(p, q) \in U_{i}$ implies $\left(p \phi^{k}, q \phi^{k}\right) \in V$ for $k= \pm i$. Since $(X, \mathscr{U})$ is not metrizable, the countable set $\left\{U_{i} \mid i=1,2, \cdots\right\}$ is not a base for the uniformity $\mathscr{U}([4]$, p. 16). Thus there exists $W \in \mathscr{U}$ with $W \subset U$ such that $i \geqq 1$ implies $U_{i} \cap$ comp $W \neq 0$. Choose, for each $i,\left(x_{i}, y_{i}\right) \in U_{i} \cap$ comp $W$. Since $X \times X$ is a compact Hausdorff space, there exists $(x, y)$ such that each neighborhood of $(x, y)$ contains $\left(x_{i}, y_{i}\right)$ for an infinite number of positive integers $i$. Let $n$ be an arbitrary positive integer, then there exists $m>n$ such that $\left(x_{m}, y_{m}\right) \in U_{n}(x) \times U_{n}(y)$. Hence $\left(x, x_{m}\right) \in U_{n}$ and $\left(y, y_{m}\right) \in U_{n}$;

Received October 3, 1959, and in revised form, February 1, 1960. 
therefore $\left(x \phi^{k}, x_{m} \phi^{k}\right) \in V$ and $\left(y \phi^{k}, y_{m} \phi^{k}\right) \in V$ for $k= \pm n$. Also $\left(x_{m}, y_{m}\right) \in$ $U_{m} \subset U_{n}$ so that $\left(x_{m} \phi^{k}, y_{m} \phi^{k}\right) \in V$ for $k= \pm n$. Hence $\left(x \phi^{k}, y \phi^{k}\right) \in V \circ V \circ V \subset U$ for $k= \pm n$. Each $\left(x_{i}, y_{i}\right) \in U_{i} \subset V$ and $\bar{V} \subset U$; hence $(x, y) \in U$. Finally, $x \neq y$. For otherwise we could choose $S \in \mathscr{C}$ such that $S \circ S \subset W$; then $\left(x_{k}, y_{k}\right) \in S(x) \times S(x)$ for some $k$, and hence $\left(x, x_{k}\right) \in S,\left(x, y_{k}\right) \in S$ so that $\left(x_{k}, y_{k}\right) \in W$. This completes the proof.

An immediate consequent of the theorem is the following

CoRollary. If $(X, \mathscr{C})$ is a compact uniform space on which it is possible to define an expansive homeomorphism, then $\left(X, \mathscr{C}^{C}\right)$ is metrizable.

3. The author is indebted to the referee for suggesting the arrangement of the material in this section. In the original version, Lemma 2 had a slightly stronger hypothesis and Lemma 3 was essentially contained in the proof of Theorem 2. In this section we suppose that $X$ is an infinite compact metric space and (with the exception of Lemma 3) that $\phi$ is an expansive homeomorphism (with expansive constant $d$ ) of $X$ onto $X$.

Lemma 1. If $x \neq y$ and if there is an integer $N$ such that $n>N$ $\{n<N\}$ implies $\rho\left(x \phi^{n}, y \phi^{n}\right) \leqq d$, then $x$ and $y$ are positively \{negatively\} asymptotic under $\phi$.

Proof. If $x$ and $y$ are not positively asymptotic under $\phi$, then there exist $\varepsilon>0$ and positive integers $n_{1}<n_{2}<\cdots$ such that $\rho\left(x \phi^{n_{i}}, y \phi^{n_{i}}\right) \geqq \varepsilon$ with $\lim _{i \rightarrow+\infty} x \phi^{n_{i}}=u$ and $\lim _{i \rightarrow+\infty} y \phi^{n_{i}}=v$. Obviously $u \neq v$. Let $m$ be an arbitrary integer. For all $i$ sufficiently large $n_{i}+m>N$; hence $\rho\left(x \phi^{n_{i}+m}, y \phi^{n_{i}+m}\right) \leqq d$. Since $\lim _{i \rightarrow+\infty} x \phi^{n_{i}+m}=u \phi^{m}$ and $\lim _{i \rightarrow+\infty} y \phi^{n_{i}+m}=v \phi^{m}$, it is clear that $\rho\left(u \phi^{m}, v \phi^{m}\right) \leqq d$ for each integer $m$. This contradicts the hypothesis that $\phi$ is expansive. The alternative statement may be proved by a similar argument.

Lemma 2. If $\omega(x)\{\alpha(x)\}$ contains a periodic point $p$ and $\omega(x)\{\alpha(x)\}$ is not identical with the orbit of $p$, then there exist $w$ and $z$ in $\omega(x)$ $\{\alpha(x)\}$ such that $w$ and $p$ are positively asymptotic and $z$ and $p$ are negatively asymptotic.

Proof. Suppose $p$ is of period $k$. There exist positive integers $n_{1}<n_{2}<\cdots$ such that $\lim _{i \rightarrow+\infty} x \phi^{n_{i}}=p$. Let $k_{i}$ be the smallest nonnegative integer such that $n_{i}+k_{i}$ is a multiple of $k$. Since $0 \leqq k_{i}<k$, there exists $m$ such that $k_{i}=m$ for an infinite number of integers $i$. Thus there are positive integers $m_{1}<m_{2}<\cdots$ such that 


$$
\lim _{i \rightarrow+\infty} x \phi^{m_{i}+m}=\lim _{i \rightarrow+\infty} x \phi^{k j_{i}}=p \phi^{m} .
$$

Denote $\phi^{k}$ by $\theta$ (with expansive constant $d_{1}$ ) and $p \phi^{m}$ by $q$ (see [1], p. 86). Thus $\lim _{i \rightarrow+\infty} x \theta^{j_{i}}=q$ and $q \theta=q$. We can assume that $\rho\left(x \theta^{j_{i}}, q\right)<d_{1}$ for each $i$.

The points $x$ and $q$ are not positively asymptotic under $\theta$, since otherwise $\omega(x)$ under $\phi$ would consist of the $k$ points in the orbit of $p$. Hence, by Lemma 1 , there exist arbitrarily large integers $r$ such that $\rho\left(x \theta^{r}, q\right)>d_{1}$. Therefore we can assume that $s_{1}<s_{2} \cdots$ are positive integers where $s_{i}$ is the smallest positive integer such that $\rho\left(x \theta^{j_{i}+s_{i}}, q\right)>d_{1}$ and $\lim _{i \rightarrow+\infty} x \theta^{j_{i}+s_{i}}=$ $u \in \omega(x)$. Let $-a$ be an arbitrary negative integer, then for all $i$ sufficiently large $0<s_{i}-a<s_{i}$. Hence $\rho\left(x \theta^{j_{i}+s_{i}-a}, q\right) \leqq d_{1}$, and therefore $\rho\left(u \theta^{-a}, q\right) \leqq d_{1}$ for each negative integer $-a$. Thus, by Lemma $1, u$ is negatively asymptotic to $q$ under $\theta$ and hence under $\phi([1], p .85)$. We can assume $j_{i}<j_{i}+s_{i}<j_{i+1}$ and hence that there exist positive integers $t_{2}<t_{3}<\cdots$ where $t_{i}$ is the smallest positive integer such that $\rho\left(x \theta^{j_{i}-t_{i}}, q\right)>d_{1}$ and $\lim _{i \rightarrow+\infty} x \theta^{j_{i}-t_{i}}=v \in \omega(x)$. By an argument similar to the above, $v$ is positively asymptotic to $q$ under $\phi$. Since $\alpha(x)$ under $\phi$ coincides with $\omega(x)$ under $\phi^{-1}$, this completes the proof.

In the following lemma we do not require $\phi$ to be expansive.

Lemma 3. If $x$ is not periodic and $\omega(x)\{\alpha(x)\}$ is the orbit of a periodic point $p$, then there exists a point $q$ in the orbit of $p$ such that $q$ and $x$ are positively \{negativoly\} asymptotic.

Proof. Let $p \in \omega(x)$ and, as in the first paragraph of the proof of Lemma 2 , select positive integers $j_{1}<j_{2}<\cdots$ such that $\lim _{i \rightarrow+\infty} x \theta^{j_{i}}=$ $q=p \phi^{m}$ and $q \theta=q, \theta=\phi^{k}$. If $x$ and $q$ are not positively asymptotic under $\theta$, then there exists a positive constant $\alpha$ and a sequence $n_{1}<n_{2}<\cdots$ of integers such that $\rho\left(x \theta^{n_{i}}, q\right)>\alpha$. Let $\varepsilon>0$ and choose $\beta>0$ such that $\beta<\varepsilon, \beta<\alpha$, and $\rho(z, w) \leqq \beta$ implies $\rho(z \theta, w \theta)<\varepsilon$. We can assume that $\rho\left(x \theta^{j}, q\right)<\beta$. Let $s_{i}$ be the smallest positive integer such that $\rho\left(x \theta^{j_{i}+s_{i}}, q\right)>\beta$. Then for each $i, \beta<\rho\left(x \theta^{j_{i}+s_{i}}, q\right)<\varepsilon$. But the sequence $\left\{x \theta^{j_{i}+s_{i}}\right\}$ has a convergent subsequence. Let $s$ be the limit of such a convergent subsequence, then $s \neq q, s \in \omega(x)$ and $\rho(s, q) \leqq \varepsilon$. Thus $\omega(x)$ is not finite, contrary to hypothesis. It follows that $x$ and $q$ are positively asymptotic under $\theta$, and hence under $\phi$.

Similarly, if $\alpha(x)$ is the orbit of a periodic point $p$, then there exists a point $q$ in the orbit of $p$ such that $q$ and $x$ are negatively asymptotic under $\dot{\phi}$.

TheOREM 2. There exist $a, b, c, d \in X$ such that $a$ and $b$ are positively asymptotic under $\phi$ and $c$ and $d$ are negatively asymptotic under $\phi$. 
Proof. There exists a minimal set $N \subset X([1], \mathrm{p} .15)$. If $N$ is infinite, then $N$ is self-dense and the conclusion follows from Theorem 10.36 of [1]. Henceforth, suppose each minimal set in $X$ is finite and thus is a periodic orbit.

Since $X$ is compact and infinite, there exists a non-isolated point $r$. If $r$ is not periodic, let $r=p$. If $r$ is periodic, then there exists $x \neq r$ such that $x$ and $r$ are asymptotic ([1], p. 87). But then $x$ is not periodic and we let $x=p$.

There exists a minimal set $N \subset \omega(p)$, and a minimal set $M \subset \alpha(p)$. Both $N$ and $M$ are periodic orbits. If $N \neq \omega(p)$ or $M \neq \alpha(p)$ the conclusion of the theorem follows from Lemma 2. If $N=\omega(p)$ and $M=\alpha(p)$, the conclusion of the theorem follows from Lemma 3.

4. In addition to the standing hypothesis of $\S 3$ we require $X$ to be self-dense.

Lemma 4. If $y \in U(\varepsilon, x)$ implies that each neighborhood of $y$ contains $z$ such that $\rho\left(y \phi^{n}, z \phi^{n}\right)>d / 2$ for some positive \{negative\} $n$, then there exists $w \in U(\varepsilon, x)$ such that $w$ and $x$ are not positively \{negatively\} asymptotic.

Proof. Let $0<\alpha<\varepsilon$, then there exist $x_{1} \in U(\alpha, x)$ and a positive integer $n_{1}$ such that $\rho\left(x_{1} \phi^{n_{1}}, x \phi^{n_{1}}\right)>d / 2$. Suppose $x_{1}$ and $x$ are positively asymptotic (otherwise the lemma holds); hence there exists $m_{1}>n_{1}$ such that $n>m_{1}$ implies $\rho\left(x_{1} \phi^{n}, x \phi^{n}\right)<d / 8$. Choose $\alpha_{1}>0$ such that $U\left(\alpha_{1}, x_{1}\right) \subset$ $U(\alpha, x)$ and $\rho(p, q)<\alpha_{1}$ implies $\rho\left(p \phi^{n}, p \phi^{n}\right)<d / 8$ for $0 \leqq n \leqq m_{1}$. For $i>1$ we select $x_{i}, n_{i}, m_{i}$, and $\alpha_{i}>0$ such that $x_{i} \in U\left(\alpha_{i-1}, x_{i-1}\right), n_{i}>m_{i-1}$, $\rho\left(x_{i} \phi^{n_{i}}, x_{i-1} \phi^{n_{i}}\right)>d / 2, m_{i}>n_{i}, n>m_{i}$ implies $\rho\left(x_{i} \phi^{n}, x \phi^{n}\right)<d / 8, U\left(\alpha_{i}, x_{i}\right) \subset$ $U\left(\alpha_{i-1}, x_{i-1}\right)$, and $\rho(p, q)<\alpha_{i}$ implies $\rho\left(p \phi^{n}, q \phi^{n}\right)<d / 8$ for $0 \leqq n \leqq m_{i}$. We can suppose $\lim _{i \rightarrow+\infty} x_{i}=w \in \overline{U(\alpha, x)} \subset U(\varepsilon, x)$ and $w \neq x$. If $i>1$, then $n_{i}>m_{i-1}$ and hence $\rho\left(x_{i-1} \phi^{n_{i}}, x \phi^{n_{i}}\right)<d / 8$. But $\rho\left(x_{i} \phi^{n_{i}}, x_{i-1} \phi^{n_{i}}\right)>d / 2$, and the triangle inequality implies $\rho\left(x_{i} \phi^{n_{i}}, x \phi^{n_{i}}\right)>3 d / 8$. If $j>i$, then $x_{j} \in U\left(\alpha_{i}, x_{i}\right)$ and, since $m_{i}>n_{i}, \rho\left(x_{j} \phi^{n_{i}}, x_{i} \phi^{n_{i}}\right)<d / 8$. Therefore $\rho\left(x_{j} \phi^{n_{i}}, x \phi^{n_{i}}\right)>d / 4$ for $j \geqq i$. If $i>1$ is fixed, then $\rho\left(x_{j} \phi^{n_{i}}, w \phi^{n_{i}}\right)$ is arbitrarily small for $j$ sufficiently large. Hence $\rho\left(x \phi^{n_{i}}, w \phi^{n_{i}}\right) \geqq d / 4$. Since $\left\{n_{i}\right\}$ is an increasing sequence of positive integers, $w$ and $x$ are not positively asymptotic. This proof establishes the alternative statement by using $\phi^{-1}$ rather than $\phi$.

THeOREM 3. For each $x \in X$ and each $\varepsilon>0$ there exists $y \in U(\varepsilon, x)$ such that $x$ and $y$ are not doubly asymptotic.

Proof. Suppose there exist $x \in X$ and $\varepsilon>0$ such that $z \in U(\varepsilon, x)$ implies $x$ and $z$ are positively asymptotic. Suppose $\varepsilon<d / 2$, then, by 
the above lemma, there exist $y \in U(\varepsilon, x)$ and $\alpha>0$ such that $U(\alpha, y) \subset$ $U(\varepsilon, x)$ and $t \in U(\alpha, y)$ implies that $\rho\left(t \phi^{n}, y \phi^{n}\right) \leqq d / 2$ for $n \geqq 0$. Therefore $u, v \in U(\alpha, y)$ implies $\rho\left(u \phi^{n}, v \phi^{n}\right) \leqq d$ for $n \geqq 0$. Thus, since $\phi$ is expansive, $u, v \in U(\alpha, y)$ implies $\rho\left(u \phi^{n}, v \phi^{n}\right)>d$ for some negative $n$. By the alternative form of the lemma above, there exists $w \in U(\alpha, y)$ such that $w$ and $y$ are not negatively asymptotic. Therefore either $w$ and $x$ are not negatively asymptotic or $y$ and $x$ are not negatively asymptotic, which establishes the theorem.

If $X$ is an infinite minimal set, then a stronger statement can be made. Since $X$ is pointwise almost periodic under $\phi([1], p .31), \varepsilon>0$ implies $\rho\left(x, x \phi^{n}\right)<\varepsilon$ for some $n \neq 0$. It is easy to show that $x$ and $x \phi^{n}$ are neither positively nor negatively asymptotic.

If $X$ is not self-dense, then, as shown by the following example, each pair of distinct points may be both positively and negatively asymptotic. Let $X$ consist of the real numbers $0,1 / n\{n= \pm 1, \pm 2, \cdots\}$, and let

$$
x \phi=\left\{\begin{array}{l}
0 \text { if } x=0 \\
1 /(n+1) \text { if } x=1 / n \text { and } n \neq-1 \\
1 \text { if } x=-1
\end{array}\right.
$$

\section{REFERENCES}

1. W. H. Gottschalk and G. A. Hedlund, Topological dynamics, Amer. Math. Soc. Colloquium Publications, vol. 36, Providence, 1955.

2. J. L. Kelley, General topology, D. Van Nostrand Co., Inc., New York, 1955.

3. W. R. Utz, Unstable homeomorphisms, Proc. Amer. Math. Soc. 1 (1950), 769-774.

4. A. Weil, Sur les espaces a structure uniform et sur la topologie générale, Actualités Sci. Ind. 551, Paris, 1937.

VANDERBILT UNIVERSITY 



\section{PACIFIC JOURNAL OF MATHEMATICS}

\section{EDITORS}

David GILbarg

Stanford University

Stanford, California

\section{F. H. BRowneLL}

University of Washington

Seattle 5, Washington

\section{A. L. Whiteman}

University of Southern California Los Angeles 7, California

\section{J. PAIGe}

University of California

Los Angeles 24, California

\section{ASSOCIATE EDITORS}
E. F. BECKENBACH
T. M. CHERRY
D. DERRY

\author{
E. HEWITT \\ A. HORN \\ L. NACHBIN
}

\author{
M. OHTSUKA \\ H. L. ROYDEN \\ M. M. SCHIFFER
}

E. SPANIER

E. G. STRAUS

F. WOLF

\section{SUPPORTING INSTITUTIONS}

\author{
UNIVERSITY OF BRITISH COLUMBIA \\ CALIFORNIA INSTITUTE OF TECHNOLOGY \\ UNIVERSITY OF CALIFORNIA \\ MONTANA STATE UNIVERSITY \\ UNIVERSITY OF NEVADA \\ NEW MEXICO STATE UNIVERSITY \\ OREGON STATE COLLEGE \\ UNIVERSITY OF OREGON \\ OSAKA UNIVERSITY \\ UNIVERSITY OF SOUTHERN CALIFORNIA
}

\author{
STANFORD UNIVERSITY \\ UNIVERSITY OF TOKYO \\ UNIVERSITY OF UTAH \\ WASHINGTON STATE COLLEGE \\ UNIVERSITY OF WASHINGTON \\ AMERICAN MATHEMATICAL SOCIETY \\ CALIFORNIA RESEARCH CORPORATION \\ HUGHES AIRCRAFT COMPANY \\ SPACE TECHNOLOGY LABORATORIES \\ NAVAL ORDNANCE TEST STATION
}

\footnotetext{
Mathematical papers intended for publication in the Pacific Journal of Mathematics should be typewritten (double spaced), and the author should keep a complete copy. Manuscripts may be sent to any one of the four editors. All other communications to the editors should be addressed to the managing editor, L. J. Paige at the University of California, Los Angeles 24, California.

50 reprints per author of each article are furnished free of charge; additional copies may be obtained at cost in multiples of 50 .
}

The Pacific Journal of Mathematics is published quarterly, in March, June, September, and December. The price per volume (4 numbers) is $\$ 12.00$; single issues, $\$ 3.50$. Back numbers are available. Special price to individual faculty members of supporting institutions and to individual members of the American Mathematical Society: $\$ 4.00$ per volume; single issues, $\$ 1.25$.

Subscriptions, orders for back numbers, and changes of address should be sent to Pacific Journal of Mathematics, 2120 Oxford Street, Berkeley 4, California.

Printed at Kokusai Bunken Insatsusha (International Academic Printing Co., Ltd.), No. 6, 2-chome, Fujimi-cho, Chiyoda-ku, Tokyo, Japan.

PUBLISHED BY PACIFIC JOURNAL OF MATHEMATICS, A NON-PROFIT CORPORATION

The Supporting Institutions listed above contribute to the cost of publication of this Journal, but they are not owners or publishers and have no responsibility for its content or policies. 


\section{Pacific Journal of Mathematics}

\section{Vol. 10, No. $4 \quad$ December, 1960}

M. Altman, An optimum cubically convergent iterative method of inverting a linear bounded operator in Hilbert space . . . . . . . . . . . . . . . . . . . . . . . . . . 1107

Nesmith Cornett Ankeny, Criterion for rth power residuacity ................. 1115

Julius Rubin Blum and David Lee Hanson, On invariant probability measures I . . . . . 1125

Frank Featherstone Bonsall, Positive operators compact in an auxiliary topology ..... 1131

Billy Joe Boyer, Summability of derived conjugate series . . . . . . . . . . . . . . . . 1139

Delmar L. Boyer, A note on a problem of Fuchs . . . . . . . . . . . . . . . . . 1147

Hans-Joachim Bremermann, The envelopes of holomorphy of tube domains in infinite

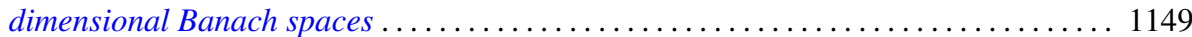

Andrew Michael Bruckner, Minimal superadditive extensions of superadditive

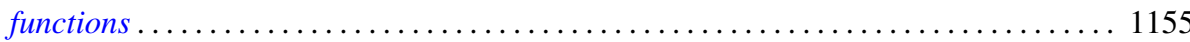

Billy Finney Bryant, On expansive homeomorphisms .................... 1163

Jean W. Butler, On complete and independent sets of operations in finite algebras . . . . . 1169

Lucien Le Cam, An approximation theorem for the Poisson binomial distribution ...... 1181

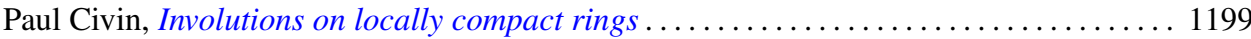

Earl A. Coddington, Normal extensions of formally normal operators . . . . . . . . . 1203

Jacob Feldman, Some classes of equivalent Gaussian processes on an interval ........ 1211

Shaul Foguel, Weak and strong convergence for Markov processes . . . . . . . . . . . 1221

Martin Fox, Some zero sum two-person games with moves in the unit interval ........ 1235

Robert Pertsch Gilbert, Singularities of three-dimensional harmonic functions . . . . . . . 1243

Branko Grünbaum, Partitions of mass-distributions and of convex bodies by

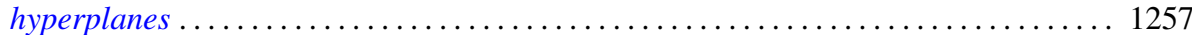

Sidney Morris Harmon, Regular covering surfaces of Riemann surfaces ........... 1263

Edwin Hewitt and Herbert S. Zuckerman, The multiplicative semigroup of integers

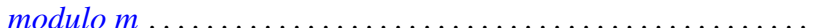

Paul Daniel Hill, Relation of a direct limit group to associated vector groups . ......... 1309

Calvin Virgil Holmes, Commutator groups of monomial groups . .

James Fredrik Jakobsen and W. R. Utz, The non-existence of expansive homeomorphisms

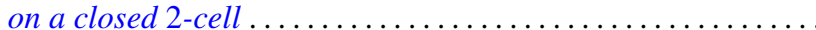

John William Jewett, Multiplication on classes of pseudo-analytic functions . . . . . . . 1323

Helmut Klingen, Analytic automorphisms of bounded symmetric complex domains . . . . 1327

Robert Jacob Koch, Ordered semigroups in partially ordered semigroups . . . . . . . . 1333

Marvin David Marcus and N. A. Khan, On a commutator result of Taussky and

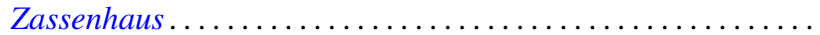

John Glen Marica and Steve Jerome Bryant, Unary algebras......

Edward Peter Merkes and W. T. Scott, On univalence of a continued fraction . . . . . . . 1361

Shu-Teh Chen Moy, Asymptotic properties of derivatives of stationary measures . . . . . 1371

John William Neuberger, Concerning boundary value problems . . . . . . . . . . . 1385

Edward C. Posner, Integral closure of differential rings . . . . . . . . . . . . . . . . . 1393

Marian Reichaw-Reichbach, Some theorems on mappings onto . . . . . . . . . . . . . 1397

Marvin Rosenblum and Harold Widom, Two extremal problems . . . . . . . . . . . . . . . . 1409

Morton Lincoln Slater and Herbert S. Wilf, A class of linear differential-difference

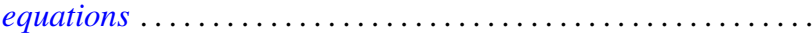

Charles Robson Storey, Jr., The structure of threads . . . . . . . . . . . . . . . . . . 1429

J. François Treves, An estimate for differential polynomials in $\partial / \partial z_{1},, \cdots, \partial / \partial z_{-} n \ldots \ldots 1447$

J. D. Weston, On the representation of operators by convolutions integrals . . . . . . . . 1453

James Victor Whittaker, Normal subgroups of some homeomorphism groups ......... 1469 